\title{
El camino de la resiliencia: del sujeto individual al sujeto político
}

The Path of Resilience: from the Individual Subject to the Political Subject

Le chemin de la résilience: du sujet individuel au sujet politique

O caminho da resiliência: do sujeito individual ao sujeito político

Fecha de recepción: 27 DE ENERO DE 2016/Fecha de aceptación: 25 DE JULIO DE 2017/Fecha de disponibilidad en línea: 1 DE DICIEMBRE DE 2017

Encuentre este artículo en http://magisinvestigacioneducacion.javeriana.edu.co/

doi: 10.11144/Javeriana.m10-20.crsi

Escrito por Luis Fernando Granados-Ospina, S.J.

Pontificia Universidad JaVeriana Cali, Colombia

Ifgranados@javerianacali.edu.co

Sara Victoria Alvarado-Salgado CINDE-Universidad de Manizales Manizales, Colombia doctoradoumanizales@cinde.org.co

JAIME CARMONA-PARRA

\section{Resumen}

Este artículo de revisión parte de algunas definiciones de la categoría de resiliencia e identifica en ellas diversas tendencias y enfoques. Desde una valoración de la experiencia humana y de la significación que tienen las interacciones sociales y las acciones comunitarias para la construcción de la resiliencia, el artículo permite explorar las fronteras donde la resiliencia se encuentra con la política, entendida en el sentido amplio como acción colectiva. La respuesta resiliente buscaría configurar sentidos y prácticas personales y comunitarias para afrontar la adversidad, desde una acción política comprometida con la dignidad humana. Los métodos utilizados son una revisión documental y un análisis categorial, que buscan ofrecer elementos teóricos para poner en diálogo la resiliencia con la política.

\section{Palabras clave}

Resiliencia; jóvenes; política; acción comunitaria

Para citar este artículo / To cite this article / Pour citer cet article / Para citar este artigo

Granados-Ospina, S.J., Luis Fernando; Alvarado-Salgado, Sara Victoria \& Carmona-Parra, Jaime (2017). El camino de la resiliencia: del sujeto individual al sujeto político. magis, Revista Internacional de Investigación en Educación, 10 (20), 49-68. https://doi.org/10.11144/Javeriana.m10-20.crsi 
Keywords

Resilience; young people; politics; community action
Mots clés

Résilience; jeunes; politique;

action communautaire

\section{Abstract}

This is a literature review that starts from some definitions of the category of resilience, identifying in them diverse tendencies and approaches. From an assessment of human experience and the significance of social interactions and community actions for the construction of resilience, the article allows us to explore the frontiers where resilience meets politics, understood in the broad sense as collective action. The resilient response would seek to configure personal and community senses and practices to face adversity, from a political action committed to human dignity. The method used is a literature review and a categorical analysis, which seeks to offer theoretical elements to put the resilience in dialogue with politics.

\section{Résumé}

Article de synthèse qui part des définitions de la catégorie de résilience, pour identifier dans celle-ci les différentes tendances et perspectives. A partir d'une appréciation de l'expérience humaine et de la signification qui ont les interactions sociales et les actions communautaires pour la construction de la résilience, l'article permet explorer les lisières où la résilience se trouve avec la politique, en tant qu'action collective; c'està-dire dans sa signification la plus ample. La réponse résiliente cherche façonner les sens et les pratiques personnelles et communautaires pour faire face à l'adversité, à partir d'une action politique engage avec la dignité humaine. La méthode utilisée est une révision documentaire et une analyse de catégories, qui vise à fournir les éléments théoriques pour mettre en place un dialogue entre la résilience et la politique.

\section{Palavras-chave}

Resiliência; jovens; política; ação comunitária

\section{Resumo}

Artigo de revisão que parte de algumas definições da categoria resiliência, identificando nelas diversas tendências e enfoques. Desde uma avaliação da experiência humana e da significação que têm as interações sociais e as ações comunitárias para a construção da resiliência, o artigo permite explorar as fronteiras onde a resiliência se encontra com a política, entendida no sentido amplo como ação coletiva. A resposta da resiliência procuraria configurar sentidos e práticas pessoais e comunitárias para enfrentar a adversidade, desde uma ação política comprometida com a dignidade humana. O método utilizado é uma revisão documental e uma análise categorial, que procura oferecer elementos teóricos para pôr em diálogo resiliência y política. 


\section{Introducción}

Este artículo parte de una mirada crítica sobre algunas definiciones de resiliencia, para reconocer el valor que estos acercamientos conceptuales tienen en la comprensión de los tránsitos de la categoría desde una visión más individualista centrada en el sujeto heroico, hacia una visión más social y política centrada en el sujeto histórico. Aquí se valoran los procesos de socialización e interacción, como lugares experienciales donde los sujetos afrontan la adversidad con otros y desde otros. Estos sujetos son actores sociales situados históricamente, que habitan territorios complejos donde se cruzan intereses y búsquedas, realidades de inequidad e injusticia, como la misma construcción social de la resiliencia que propugna por respuestas creativas a los retos que hoy enfrenta la sociedad en un contexto globalizado.

Origen de la categoría de resiliencia: primera y segunda generación de investigadores

Es notable que la Real Academia de la Lengua en la 23ª edición (2014) de su diccionario haya incorporado la palabra resiliencia y aunque la definición presentada no dé cuenta del constructo en su complejidad y dinamismo, tiene el valor de ofrecer un reconocimiento y visibilidad a una palabra que, en su uso, se ha ido incorporando en la cotidianidad del lenguaje, para nombrar los tránsitos que experimentan los sujetos y las comunidades a partir del afrontamiento de situaciones de adversidad.

Resiliencia. Del ingl. resilience, y este der. del lat. resiliens, -entis, part. pres. act. de resilīre 'saltar hacia atrás, rebotar', 'replegarse'. 1. f. Capacidad de adaptación de un ser vivo frente a un agente perturbador o un estado o situación adversos. 2. f. Capacidad de un material, mecanismo o sistema para recuperar su estado inicial cuando ha cesado la perturbación a la que había estado sometido (Real Academia Española, RAE, 2014).

En el campo de las Ciencias Sociales, los estudios en resiliencia han ido cobrando una gran relevancia, como demuestra la producción de trabajos de grado en las universidades colombianas en la última década (Campo-Vásquez, Granados-Ospina, S.J., Muñoz-Ortega, Rodríguez-Arenas \& Trujillo-García, 2012). Tal interés está ocupando a psicólogos, médicos, sociólogos, antropólogos, educadores, ecologistas y trabajadores sociales, entre otros, que han venido encontrando en la resiliencia una categoría que en su reflexión va dando luces para comprender cómo los sujetos y las comunidades se transforman y trabajan por una vida digna, en medio de situaciones de adversidad.

La resiliencia se presenta, efectivamente, como un verdadero disfraz de Arlequín. Las numerosas disciplinas que la consideran como objeto de estudio y los múltiples paradigmas en los que se inscribe han contribuido a dar de ella una visión polisémica, incluso caótica. Es cierto que su complejidad es tal que ha requerido un análisis en segmentos separados mediante abordajes diferentes. Hoy en día, la tendencia es la pluridisciplinariedad y el planteamiento integrativo: genético, psíquico, medioambiental, biológico, cultural, emocional, educativo, cognitivo (Pourtois, 2014, pp. 71-72).

El término que procede del vocablo latino resilio (Kotliarenco, Cáceres \& Fontecilla, 1997) está indicando un proceso humano que tiene qué

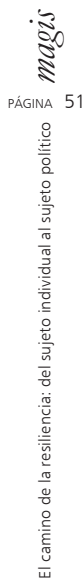

Descripción del artículo | Article description | Description de l'article | Artigo descrição

Este artículo de revisión surgió en el trabajo de investigación doctoral La resiliencia y lo político: en estudiantes vinculados al Programa "Ser Pilo Paga" de la Universidad Javeriana de Cali, que tuvo como finalidad ampliar la frontera del saber epistémico sobre la resiliencia, desde el horizonte de lo político y la política, para establecer un diálogo entre resiliencia y política. 
ver con volver atrás, resaltar y rebotar hacia adelante para salir fortalecido. La resiliencia aparece como "resistencia" a los golpes de la vida en la que acontecen conflictos y tensiones. El concepto hizo tránsito de la física a las ciencias sociales, precisamente por su flexibilidad para nombrar experiencias de vida que en sus relatos podrían integrar lo que Boris Cyrulnik llamó oxímoron: "figura retórica que consiste en reunir dos términos antinómicos" (Cyrulnik, 2001, p. 21). Según Boris Cyrulnik, el oxímoron se vuelve característico de una personalidad herida, pero resistente; sufriente, pero fuerte a pesar de todo, una persona que frente a una experiencia dura sigue tejiendo la vida con esperanza, sin dejarse destruir por la desgracia.

Por analogía, en las ciencias humanas y particularmente en la psicología se utilizó este término para dar cuenta de la capacidad humana que permite a las personas, que a pesar de atravesar situaciones adversas, dolorosas o difíciles puedan salir de ellas no solamente a salvo, sino aún enriquecidas por la experiencia (Cyrulnik, Tomkiewicz, Guénard, Vanistendael, Manciaux \& Balegno, 2004, p. 12).

Cyrulink de manera coloquial comenta cómo fue llegando casi fortuitamente al concepto, al contar la historia de unos marineros que hablaban de la resiliencia. Estos trabajadores explicaban cómo los submarinos eran sometidos a tensión, compresión, golpes, pero que, al pasar las pruebas, mostraban cierta elasticidad para volver a recuperarse. El hecho de que no se fueran al fondo y salieran de nuevo, expresaba su "resiliencia": una capacidad reconocida para recuperar su forma natural después de haber sido expuestos a fuertes presiones.

Una desgracia nunca es maravillosa. Es un fango helado, un barro negro, una escara dolorosa que nos obliga a escoger: someterse o sobreponerse. La resiliencia define el resorte de los que, habiendo recibido un golpe, han podido sobrepasarlo. El oxímoron describe el mundo íntimo de esos vencedores heridos (Cyrulnik, 2001, p. 23).

Esta cualidad resiliente de soportar golpes y salir fortalecido, adquiere mucha potencia en el ámbito de la construcción humana y social, e invita a un cambio de actitud frente al propio desarrollo humano y a la manera como los sujetos afrontan la adversidad en medio del dolor de sus heridas. Sin desconocer la complejidad de la adversidad, el concepto en sus orígenes emerge para dar cuenta de los tránsitos del sujeto para hacerse más humano en medio de sus adversidades.

En consecuencia, no se trata de decir: usted está herido, por lo tanto está perdido. Sino: usted está herido: ¿qué va a hacer con esta herida? ¿Va usted a gemir, a llorar? ¿Va usted a permanecer prisionero del pasado? ¿Va usted a buscar manera de vengarse? ¿Va usted a buscar el modo de hacer una carrera de víctima, tal como lo proponen todas las sociedades? O, por el contrario, con nuestra ayuda y su trabajo, ¿vamos los dos a comenzar a poner en obra un proceso de reparación y de reanudación del desarrollo? (Cyrulnik, Manciaux, Sánchez-Botero, Colmenares, Olaya \& Balegno, 2002, pp. 31-32).

Boris Cyrulnik, Michel Manciaux, Stefan Vanistendael y Jacques Lecomte (2003, p. 20) advierten que uno de los primeros que usó el concepto de resiliencia fue el psicólogo John Bowlby (1995). Al respecto, sostienen que Bowlby entendía la resiliencia como un: "resorte moral, cualidad de una 
persona que no se desanima, que no se deja abatir" (Cyrulnik, Manciaux, Vanistendael \& Lecomte, 2003, p. 20). Bowlby, que trabajaba en las carencias de cuidados maternales, se inspiró en la etología animal para impulsar sus estudios sobre la vinculación y fundamentar su teoría del apego en la configuración de vínculos seguros con los cuidadores, vínculos que tienden a perpetuarse y a favorecer al sujeto. Bowlby creía que el grado de respuesta frente a los hechos estresantes de la vida se hallaba sólidamente determinado por las estructuras de vinculación que el sujeto desarrollaba en sus primeros años de vida.

El apego, según Bowlby (1995), está dirigido hacia uno o algunos individuos, por lo general con un claro orden de preferencia y tiende a persistir durante una gran parte del ciclo vital. Hay una relación causal entre los vínculos establecidos con los padres o cuidadores y la capacidad futura del niño para establecer vínculos afectivos y reaccionar frente a eventos desestabilizadores. Bowlby (1995) pone la génesis de la resiliencia en las relaciones vinculares que construye el sujeto, desde los mecanismos del apego.

El punto clave de mi tesis es que existe una intensa relación causal entre las experiencias de un individuo con sus padres y su posterior capacidad para establecer vínculos afectivos y que ciertas variaciones corrientes de dicha capacidad, que se manifiestan en problemas conyugales y conflictos con los hijos, así como en síntomas neuróticos y trastornos de la personalidad, pueden atribuirse a determinadas variaciones corrientes de los modos de desempeñar los padres sus correspondientes roles (Bowlby 1995, p. 163).

Boris Cyrulnik reconoce que John Bowlby "lo único que deseaba al final de su vida era que comenzaran los trabajos sobre resiliencia" (Cyrulnik, 2001, p. 14). Cyrulnik recogió los aportes de la teoría del apego y trabajó este tema en su obra Bajo el signo del vínculo: una historia natural del apego (Cyrulnik, 2005a). En su ejercicio reflexivo sobre los complejos mecanismos de los vínculos atiende la amplia gama de señales que fundan el vínculo con la madre, muestra que solo entre los humanos hace falta algo más que la conducta de la paternidad biológica y alude a la función paternal simbólica, que está en la raíz de la existencia como seres culturales.

Cuando las gaviotas y los bebés Iloran, nos hacen comprender que toda información está escrita en lo biológico. Y cuando esta estimulación es percibida, adquiere sentido porque es interpretada. La historia de quien percibe da sentido a esa percepción. Lo biológico y lo histórico entremezclados nos permiten asistir al nacimiento del sentido (Cyrulnik, 2005a, p. 66).

No obstante, para tener una base sólida acerca del surgimiento de la resiliencia hay que remitirse a los estudios longitudinales realizados por las psicólogas estadounidenses Emmy E. Werner y Ruth S. Smith (1982, 1992, 2001) sobre infancia en situación de riesgo, al acompañar el desarrollo de más de 200 niños que pudieron sobreponerse a pesar de haber tenido una infancia difícil. Francisca Infante-Espínola (2002) reconoce que el trabajo de estas autoras fue el detonante de la preocupación futura sobre la resiliencia. Sin que ese trabajo indagara precisamente sobre esta temática, las observaciones y preguntas que suscitó tuvieron una repercusión definitiva en el surgimiento de la resiliencia como tema investigativo.

Un aspecto de la investigación realizada por Emmy E. Werner y Ruth S. Smith (1982) Ilamó poderosamente la atención: algunos niños supieron "sobreponerse" a partir de una infancia difícil. Las expectativas de fracaso fueron superadas y tuvieron unos desarrollos exitosos. Otros no desarro- 
Ilaron los trastornos previstos. Antes bien, mostraron una "admirable invulnerabilidad". La pregunta era obligada: ¿cómo explicar esta realidad? ¿Cómo argumentar el hecho de que algunos individuos resistieron mejor que otros los embates de la vida, la adversidad y la enfermedad? ¿Esto tenía una explicación?

Según la mirada de Infante-Espínola (2002), este trabajo inspiró lo que se ha llamado la primera generación de investigadores en resiliencia, venidos especialmente de Estados Unidos y Gran Bretaña. Estos académicos centraron su atención en los atributos individuales, los vínculos afectivos, el ambiente social protector de los sujetos, y en los factores diferenciadores que posibilitan las particulares respuestas de los sujetos frente a condicionamientos de entornos difíciles. A esto se lo llamó el modelo triádico de la resiliencia, que se configuraba desde los atributos personales, el ámbito familiar y los escenarios sociales donde acontece la vida de los sujetos (Infante-Espínola, 2002).

Desde un principio, las relaciones sociales amables y seguras, los vínculos afectivos y las expresiones de cariño y acogida fueron factores que protegían al sujeto como un resorte frente al futuro. La capacidad para dar y recibir afecto es la verdadera fuente de la resiliencia. Saber amar, ser solidario, acompañar al otro en su dolor, establecer lazos cálidos y serviciales es el principal factor que promueve la resiliencia:

El regreso a la vida se realiza en secreto, con el extraño placer que proporciona el sentimiento de vivir una prórroga. El trauma ha hecho añicos la personalidad anterior, y cuando nadie reúne los pedazos para frenar su dispersión, el sujeto queda muerto o no vuelve bien a la vida. Sin embargo, cuando se ve sostenido con la afectividad cotidiana de las personas que están cerca de él, y cuando el discurso cultural da sentido a su herida, consigue retomar un tipo de desarrollo distinto (Cyrulnik, 2005b, p. 21).

Este énfasis en los vínculos amorosos se hace recurrente en las primeras investigaciones. Stefan Vanistendael y Jacques Lecomte (2002) relatan en sus trabajos cómo muchos niños y jóvenes pudieron surgir y desarrollarse en contextos culturales y sociales diferentes, gracias a la presencia amorosa de alguien que les brindó en un momento de la vida una aceptación incondicional.

La resiliencia nos recuerda que todos nosotros, niños y adultos, necesitamos ser amados. El amor significa la aceptación profunda de la personalidad, su reconocimiento estable en la vida del otro, aun cuando se manifiesten comportamientos o rasgos de carácter que no se puedan aceptar (Vanistendael \& Lecomte, 2002, p. 29).
Los primeros trabajos en resiliencia se realizaron en personas con trastornos patológicos como esquizofrenia, personas expuestas al estrés y sujetos en condición de pobreza, en busca de reconocer el funcionamiento de estos frente a situaciones adversas (Cicchetti, 2003). Tanto la escuela anglosajona, como la europea han situado el tema de la resiliencia desde una perspectiva psicosocial.

Norman Garmezy (1991), en New York, Estados Unidos, ha sido uno de los pioneros en la conceptualización y el estudio de la capacidad de recuperación de los sujetos sometidos a situaciones de adversidad. Según Garmezy, la resiliencia es entendida como: "la capacidad para recuperarse, y mantener una conducta adaptativa, después del abandono o la incapacidad inicial al iniciarse un evento estresante" (Garmezy, 1991, p. 459). No obstante, uno de los asuntos difíciles de la resiliencia es su propia definición, como lo señala Michel Rutter (2012) al insistir en el carácter dinámico del concepto.

La resiliencia es un fenómeno que manifiestan sujetos jóvenes que evolucionan favorablemente, aunque hayan experimentado una forma de estrés que en la población generalmente se estima que implica un grave riesgo de consecuencias desfavorables (Rutter, 1993, p. 626).

Elisardo Becoña, psicólogo clínico y profesor de la Universidad de Santiago de Compostela, recoge en su artículo Resiliencia: definición, características y utilidades del concepto (Becoña, 2006), una buena síntesis de la primera generación de investigadores. En su trabajo se advierte que los primeros estudios en resiliencia se concentraron en individuos con esquizofrenia, en personas que habían estado sometidas a situaciones de pobreza extrema y traumas en su vida temprana y que no demostraban en su vida futura problemas psicológicos o de adaptación social.

El origen del estudio de la resiliencia en psicología y psiquiatría procede, por tanto, de los esfuerzos por conocer la etiología y el desarrollo de la psicopatología, especialmente de los niños en riesgo de desarrollar psicopatología debido a enfermedades mentales de los padres, problemas perinatales, conflictos interpersonales, pobreza o una combinación de estos factores (Becoña, 2006, p. 126).

Aquí se ve el interés de los primeros investigadores por la psicopatología, al pensar la resiliencia desde la experiencia de niños, niñas y jóvenes en situación de riesgo y vulnerabilidad, que han padecido o sufrido enormes problemas, traumas o situaciones estresantes, desgarradoras o desafiantes en su infancia y que 
han tenido la capacidad de recuperarse y de mantener una conducta normal. Por eso, no es de extrañar que las primeras definiciones de resiliencia de esta generación de investigadores preferentemente anglosajones, hablen de la resiliencia como una capacidad adaptativa que posibilita buenos resultados en los sujetos, a pesar de tener muchas y serias amenazas para su desarrollo (Garmezy, 1991).

El concepto no pretende presentar una imagen heroica de unos niños en comparación con otros que se enfrentan a situaciones similares de riesgo o desesperación. De hecho, hablar de resiliencia no refleja necesariamente una impermeabilidad al estrés. Más bien, está diseñado para reflejar la capacidad de recuperación y mantener una conducta adaptativa que puede seguir al retroceso o incapacidad al iniciar un evento estresante (Garmezy, 1991, p. 459).

Desde la mirada adaptativa se ve claro que la resiliencia requiere para su verificación una evaluación cualitativa del funcionamiento del sujeto dentro de los parámetros de lo que se acepta como normal (Masten, 1994). Tal evaluación se hace desde los criterios de normalidad establecidos e implica una visión del desarrollo normalizado en diferentes contextos culturales. La idea es poder determinar lo que se considera "apropiado" y "normal" tanto en el área cognitiva, como en conductas emocionales y sociales.

La segunda generación de investigadores fue más allá de las cualidades personales, los factores protectores, los vínculos amorosos y los procesos de "adaptabilidad" y se preguntó por las condiciones psicosociales y aquellos procesos sociales y culturales que favorecen o dificultan una respuesta del sujeto frente a la adversidad. Estos investigadores se distanciaron de una concepción de la resiliencia centrada en la herencia o en los genes del sujeto como algo constitutivo, y la consideraron como algo que está presente en las respuestas que los sujetos dan a determinadas situaciones adversas (Kalawski \& Haz, 2003).

Las preguntas de los investigadores de esta generación están centradas sobre todo en los procesos sociales y en los factores que inciden en los sujetos al tener que dar una respuesta en situaciones de dificultad, para conceptuar que en la resiliencia se suman factores individuales, familiares y sociales, unidos a factores protectores compuestos por recursos personales y sociales (Gómez \& Kotliarenco, 2010).

Entre los trabajos investigativos de esta segunda generación son muy bien valorados los de la psicóloga argentina Edith Henderson-Grotberg (2006). Esta autora postula una mirada optimista de la resiliencia y la entiende como una capacidad humana que poseen los sujetos para el afrontamiento de la adversidad. Henderson-Grotberg, desde una perspectiva psicosocial, entiende la resiliencia asociada a factores ambientales contextuales que la posibilitan:

Poseemos esa capacidad humana que nos hace resilientes, lo que nos permite poder afrontar el bombardeo de situaciones y acontecimientos estresantes. Al afrontar esos acontecimientos nos volvemos más fuertes, más seguros de nuestras capacidades, más permeables al estrés que otras personas estén experimentando y también más hábiles para producir cambios y minimizar o eliminar aquello que nos hace daño (Henderson-Grotberg, 2006, p. 10).

Como bien lo refieren Anna Forés y Jordi Grané (2008), esta segunda generación, liderada por Edith Henderson-Grotberg (2006), se concentra 
no tanto en las cualidades individuales y atributos personales, como en los procesos y los vínculos que permiten la configuración de una respuesta que articula lo propio del sujeto con los apoyos del ambiente.

La resiliencia no es una sustancia, algo hecho; más bien es una obra de tejedora que enhebra la seda de las capacidades personales con la seda afectiva y social en un proceso inacabado. Todas las personas hemos de tejer nuestra personalidad con el ganchillo de un punto de encuentro afectivo y social. Dicho con otras palabras, la resiliencia es un proceso diacrónico y sincrónico donde las fuerzas biológicas se articulan con el contexto social para posibilitar la transformación de la persona traumatizada (Forés \& Grané, 2008, p. 32).

Los mismos autores entienden la resiliencia como "una metáfora de las posibilidades" (Forés \& Grané, 2012, p. 12) y la sitúan en diálogo constructivo con escenarios socioeducativos de socialización, para transitar hacia una perspectiva holística de la resiliencia y destacan tres aspectos básicos observables según ellos en los procesos de resiliencia: la energía de relación, la energía de aprendizaje y la energía creativa. La experiencia de los vínculos y las sinergias nutrientes, los aprendizajes vitales y las formas siempre nuevas de superar las barreras y los obstáculos de la vida.

El modelo holístico de resiliencia no está orientado a desarrollar fórmulas o recetas simplificadas aplicables a distintos contextos, sino a ofrecer herramientas que puedan sacar a la luz la riqueza y profundidad de los recorridos resilientes. Precisamente a través de esta complejidad es posible desvelar dinámicas y sinergias que pueden aplicarse en la promoción de los procesos de resiliencia específicos (Forés \& Grané, 2012, p. 30).

Otros investigadores reconocidos de esta nueva generación son Nan Henderson y Mike M. Milstein (2003), que llevaron el asunto de la resiliencia a la escuela como escenario formativo, en espera de que allí se estimularan factores protectores, tanto internos como ambientales, que mitigaran el impacto de las situaciones adversas en niños, jóvenes y demás miembros de la comunidad educativa. Desde la perspectiva de estos autores, los trabajos en resiliencia desplazaron el modelo de desarrollo humano centrado en la patología y se aproximaron a un modelo constructivo basado en el trabajo compartido para un mejor vivir. El modelo propuesto desafía los diagnósticos que rotulan y clasifican enfermedades, para fijarse en las fuerzas que mantienen sana a la gente.
Todo individuo tiene una capacidad para la resiliencia que debe ser reconocida. Pueden descubrirse rasgos de resiliencia en casi cualquier persona, si se le examina en búsqueda de signos de resiliencia con la misma minuciosidad con la que se procura detectar problemas y déficit. El proceso de adquirir resiliencia es, de hecho, el proceso de la vida, dado que toda la gente debe superar episodios de estrés, trauma y rupturas en el proceso de vivir. Una actitud constructora de resiliencia en la escuela debe buscar "todo indicio" de resiliencia, rastreando ocasiones en las que tanto docentes como alumnos sortearon, superaron, sobrellevaron o vencieron la adversidad que enfrentaban (Henderson \& Milstein, 2003, p. 22).

En los trabajos de Michel Manciaux (2003), Stefan Vanistendael y Jacques Lecomte (2002) y Boris Cyrulnik (2001), investigadores de segunda generación, hace eco la pregunta que ya se habían planteado los primeros investigadores, en relación con las respuestas diferentes de sujetos que viven situaciones adversas similares. Pregunta que, en el caso de los primeros investigadores, se había respondido por la vía de la invulnerabilidad y la resistencia al estrés por parte de niños considerados por ellos como excepcionales (Garmezy, 1991).

En estos autores, la fuerza la tienen tanto el sujeto, como los vínculos y las interacciones en los diferentes escenarios donde acontecen los procesos de socialización y convivencia. La resiliencia exige una construcción social, un compartir la vida con otros. No podemos desarrollar resiliencia en el aislamiento radical. Si bien el sujeto puede salir hacia adelante con su esfuerzo personal, la resiliencia se hace más profunda con los otros.

No se puede desencadenar un proceso de resiliencia si se está solo. La "falta de sentido", la necesidad de construir relatos... El relato que hago yo mismo de lo que me ha ocurrido, el relato que haces tú de lo que me ha ocurrido y el relato que hace la cultura de lo que me ha ocurrido... Mediante la convergencia de todos estos relatos culturales es como voy a tener una representación de mí mismo. Si estás herido, quizás te van a acompañar y te ayudarán a reanudar tu desarrollo. Ahí hay posibilidad de resiliencia (Cyrulnik, 2014, pp. 33-34).

Stefan Vanistendael (2014) reconoce que no hay una única definición de resiliencia. Le apuesta a una comprensión más pragmática, al entender que la resiliencia inspira formas creativas para afrontar la vida desde una esperanza realista. La articulación de estas dos realidades estaría en el corazón, en la esencia de la resiliencia. 
A falta de definición universalmente reconocida de la resiliencia humana, proponemos una simple definición pragmática: la capacidad de una persona o de un grupo para crecer en presencia de muy grandes dificultades... la resiliencia no es fija, sino que varía a lo largo de toda la vida; nunca es absoluta; se construye en interacciones con el entorno... siempre se encuentra en proceso; y, más allá de la simple resistencia, construye o reconstruye la vida (Vanistendael, 2014, p. 53).

Desde la perspectiva de Boris Cyrulnik, Michel Manciaux, Stefan Vanistendael y Jacques Lecomte (2003), la resiliencia es un proceso de agenciamiento de la vida, un quehacer dinámico, evolutivo en los sujetos, en los que las adversidades pueden ser afrontadas desde recursos propios y apoyos del entorno.

La resiliencia es la capacidad de una persona o de un grupo para desarrollarse bien, para seguir proyectándose en el futuro a pesar de acontecimientos desestabilizadores, de condiciones de vida difíciles y de traumas a veces graves... La resiliencia nunca es absoluta, total, lograda para siempre. Es una capacidad que resulta de un proceso dinámico, evolutivo, en la que la importancia de un trauma puede superar los recursos del sujeto; varía según las circunstancias, la naturaleza del trauma, el contexto y la etapa de la vida; puede expresarse de modos muy diversos según la cultura (Cyrulnik, Manciaux, Vanistendael \& Lecomte, 2003, pp. 22-23).

Boris Cyrulnik, Michel Manciaux, Stefan Vanistendael y Jacques Lecomte (2003) mantienen una mirada crítica y realista sobre los propios límites y posibilidades de la resiliencia. Esto es notable, pues en la literatura estudiada, existe poca capacidad crítica para examinar la resiliencia, al reconocer sus límites tanto en sus fundamentos epistemológicos, teóricos y metodológicos, como en sus intencionalidades éticas, estéticas, políticas y sus usos en el ámbito de la intervención social.

Desde una concepción sistémica y después de haber trabajado con familias funcionales y en situaciones de riesgo, Froma Walsh (2004) propone una comprensión sistémica de la resiliencia en estos términos:

La resiliencia puede definirse como la capacidad de una persona para recobrarse de la adversidad fortalecida y dueña de mayores recursos. Se trata de un proceso activo de resistencia, autocorrección y crecimiento como respuesta a la crisis y desafíos de la vida (Walsh, 2004, p. 26).

Froma Walsh (2004) reconoce que es necesario poner en tensión una concepción tradicional que afirma que no es posible eliminar los efectos de los traumas graves que se han sufrido en la infancia. Esas realidades que pasan y que dejan heridas y huellas profundas difícilmente se reparan. Frente a esta visión dramática, la postura de la autora es optimista y esperanzadora:

La resiliencia implica algo más que la mera posibilidad de sobrevivir a un suplicio horrible, atravesarlo o eludirlo... Las virtudes propias de la resiliencia permiten a las personas curar sus dolorosas heridas, hacerse cargo de su vida, amar y desarrollar una existencia plena... La resiliencia se forja cuando el individuo se abre a nuevas experiencias y actúa en forma interdependiente con los demás (Walsh, 2004, p. 27).

La resiliencia se entiende aquí como una interacción entre lo innato y lo adquirido entrelazado por adecuadas relaciones de apoyo. No obstante, 
desde su comprensión sistémica, adquieren relevancia los ámbitos familiares, sociales, económicos, políticos y culturales donde acontece la vida de los sujetos y de las comunidades.

Al pensar la resiliencia familiar y con una mirada latinoamericana, se destaca el trabajo de Esteban Gómez y María Angélica Kotliarenco (2010), autores que revisaron los antecedentes de la resiliencia familiar desde sus orígenes y la ponen en diálogo con la intervención clínica y psicosocial, con familias altamente vulnerables. Llama la atención su definición de resiliencia familiar.

Proponemos que la resiliencia familiar se defina como el conjunto de procesos de reorganización de significados y comportamientos que activa una familia sometida a estrés, para recuperar y mantener niveles óptimos de funcionamiento y bienestar, equilibrar recursos y necesidades familiares, y aprovechar las oportunidades de su entorno (Gómez \& Kotliarenco, 2010, p. 124).

Boris Cyrulnik (2001) profundiza en la concepción de proceso y en el poder evocador de las narrativas en resiliencia. Cyrulnik reconoce que la acción del sujeto es vital, pues es capaz de relatar la propia historia, darle un sentido y transformarla. La idea de proceso permite entender la dinámica resiliente en función de un caminar que integra múltiples factores, unos de riesgo y otros protectores.

La resiliencia constituye un proceso natural en el que lo que somos en un momento dado necesariamente debe entretejerse con los medios ecológicos, afectivos y verbales. Basta con que uno solo de esos medios falle para que todo se hunda. Basta con que haya un solo punto de apoyo para que la edificación pueda continuar (Cyrulnik, 2001, p. 15).

María Cristina García-Vesga y Elsy Domínguez de la Ossa, en su trabajo más cercano a nosotros Desarrollo teórico de la resiliencia y su aplicación en situaciones adversas (García-Vesga \& Domínguez de la Ossa, 2013), hacen una revisión analítica de varias definiciones y coinciden en que la mayoría de los teóricos entiende la resiliencia como un proceso social e intrapsíquico que se da en las interacciones entre sujetos. Estas investigadoras reconocen que las definiciones transitan entre las que relacionan el concepto con adaptabilidad, las que introducen el término de capacidad o habilidad, las que enfatizan el nexo entre factores internos y externos y aquellas que definen la resiliencia como adaptación y proceso.

También son notables los trabajos de Gema Puig y José Luis Rubio, sobre todo en dos textos: Manual de resiliencia aplicada (2011) y Tutores de resiliencia. Dame un punto de apoyo y moveré MI mundo (2015). En el primer texto, después de un exhaustivo trabajo de documentación en el campo de la resiliencia, los autores advierten que sigue habiendo una dificultad para hablar del concepto, por la proliferación de definiciones.

Nos encontramos frente a un constructo complejo, conformado por múltiples componentes, lo cual complica su definición... La resiliencia es un concepto en continua evolución, dado que han sido muchos los autores que han propuesto una definición (Puig \& Rubio, 2011, pp. 38, 40).

Estos autores reconocen la necesidad de pensar interdisciplinarmente la resiliencia, valoran la vida cotidiana como lugar donde acontece la lucha por la supervivencia propia de la esencia humana, se sitúan en una 
visión optimista sobre la vida y valoran la búsqueda de la felicidad como un motivador para la acción en medio de la adversidad. Algo interesante es su acercamiento a la construcción social de la resiliencia, al reconocer el papel de la comunidad y las redes vinculares relacionadas con el cuidado, el apoyo y el afecto.

El nicho ecológico es la red de relaciones necesarias para la supervivencia. Y es que, gracias a la reciprocidad afectiva y comunicativa, los niños tejen su red de relaciones, en primer lugar, con sus cuidadores, que les transmiten su experiencia y los protegen de los peligros, y posteriormente con la sociedad (Puig \& Rubio, 2011, p. 125).

En el segundo libro Tutores de resiliencia. Dame un punto de apoyo y moveré MI mundo (2015), un texto escrito en un lenguaje vivencial, los autores reconocen la significación que para la resiliencia tienen los nichos sensoriales, los puntos de apoyo, los vínculos, las interacciones, los encuentros, la presencia de los otros en tiempos de crisis y el fortalecimiento de los vínculos, como potentes mediadores para suscitar la resiliencia.

Cyrulnik define el encuentro con el tutor como algo fugaz. Un encuentro significativo puede ser suficiente. $Y$, aunque podría pensarse que, al tratarse de un momento tan efímero, algo tan aparentemente insignificante, el efecto fuese mínimo, un encuentro casual de estas características, en determinados momentos se constituye como el detonante de la recuperación, como la chispa que reaviva los rescoldos. Por tanto, con una capacidad reconstructora y regeneradora como para impulsar un proceso de resiliencia (Puig \& Rubio, 2015, p. 139).

Aunque el encuentro sea casual o anecdótico, no lo es necesariamente la fuerza de la representación que de él se tiene. El recuerdo del encuentro pervive y sigue aconteciendo como dinamismo transformador que impulsa al sujeto a seguir tejiendo su vida en medio de la adversidad. Desde este horizonte relacional se entiende la fuerza que los autores les dan a la dimensión comunitaria y social de la resiliencia y a aquellas redes vinculares que se vuelven significativas en un momento particular de la vida. Por eso no se cansan de insistir en la construcción dialógica de los procesos de vida, que le da a la resiliencia un claro sentido comunitario y social.

El concepto de resiliencia no es un atributo personal, nos remite a la importancia de la imbricación de la persona en su matriz social... es falso el dilema establecido entre la aportación personal y la aportación social. Sabemos que las dos cosas están presentes en la resiliencia, y la dosis dependerá de cada caso... podemos afirmar que la dimensión comunitaria es una condición sine qua non (Forés \& Grané, 2008, pp. 34-35).

El concepto además está reclamando un abordaje interdisciplinar que aporte miradas diferentes que lo enriquezcan (Madariaga, Palma-García, Surjo, Villalba \& Arribillaga, 2014). Esto es precisamente lo que había constatado Michel Delage (2010) al reconocer que, para comprender la resiliencia, se ha de recurrir a campos de conocimiento heterogéneos que permitan abarcar especialmente los aspectos relacionales y contextuales. Según la perspectiva de Delage (2010), la resiliencia solo puede comprenderse desde una mirada más compleja y sistémica. 
La otra corriente, que podemos calificar de holista o global, sostiene, que ciertos fenómenos solo pueden comprenderse en una perspectiva concentrada en la complejidad, es decir, en la manera en la que se intersecan los diferentes niveles, los diferentes sistemas para producir consecuencias que no pueden reducirse al análisis de las partes separadas. Evidentemente, en el caso de la resiliencia, debe prevalecer esta segunda actitud, pues se trata de una noción compleja y que, por lo tanto, requiere de diferentes niveles de abordaje (Delage, 2010, p. 90).

\section{Ámbito latinoamericano: la resiliencia y su dimensión comunitaria, social y política}

En el ámbito latinoamericano, las investigaciones en resiliencia se han concentrado más en la aplicabilidad del concepto, mediante modelos y programas de intervención que manifiestan un interés más práctico, en la búsqueda de un fortalecimiento de las prácticas de niños, mujeres y familias, en contextos socioculturales específicos (Álvarez \& Hurley, 2010). En estos trabajos investigativos y de intervención social, se busca poder entender cómo algunas comunidades, o grupos sociales, incluso ciudades y pueblos enteros, se pueden reponer propositivamente, en medio de calamidades desgarradoras, fuertes catástrofes naturales o en circunstancias agobiantes de violencia social, económica y política.

Sin duda, desde la perspectiva comunitaria de la resiliencia, hay que reconocer el aporte del Centro de Estudios y Atención al Niño y la Mujer, CEANIM, organización no gubernamental que, por más de 34 años en Chile, se ha orientado al estudio, diseño y ejecución de programas de desarrollo humano, comprometidos con la realidad de personas que viven en situación de pobreza y de desventajas sociales, económicas y culturales desde el horizonte de la resiliencia. Desde la visión de su directora María Angélica Kotliarenco, la resiliencia se puede definir de la siguiente manera:

De acuerdo a los procesos reflexivos realizados, podemos definir la resiliencia como un proceso dinámico, de origen interactivo y sociocultural que conduce a la optimización de los recursos humanos y permite sobreponerse a las situaciones adversas, gracias a la defensa que le brindan los mecanismos protectores, sean estos individuales, familiares, comunitarios y/o culturales (Kotliarenco, 2014. p. 163).

Esta autora y otros investigadores muy reconocidos, como Aldo Melillo y Elbio Néstor Suárez-Ojeda publicaron Actualizaciones en resiliencia (Kotliarenco, Mardones, Melillo \& Suárez, 2000). En este trabajo se puso de relieve la cercanía de la resiliencia con los procesos de desarrollo infantil, se revisaron algunos fundamentos psicológicos del concepto, algunas particularidades metodológicas en los estudios sobre resiliencia y se introdujo propiamente la concepción de la resiliencia desde América Latina. De sus interesantes aportes en cuanto a la comprensión del concepto de resiliencia podemos destacar lo siguiente:

La resiliencia se produce en función de procesos sociales e intrapsíquicos. No se nace resiliente ni se adquiere "naturalmente" en el desarrollo: depende de ciertas cualidades del proceso interactivo del sujeto con los otros humanos, responsable de la construcción del sistema humano (Melillo, Estamatti \& Cuestas, 2000, p. 28).

En sintonía con estos ejercicios reflexivos, es notable el trabajo que realizó María Eugenia Colmenares (2002a, 2002b) con otros académicos sensibles al tema de la resiliencia en Colombia. Sus reflexiones pusieron en tensión la comprensión de la resiliencia con los fenómenos sociales y políticos del país pasados por las violencias, las múltiples pobrezas y la inequidad.

Plantear la resiliencia como instrumento teórico de interrogación sobre la coyuntura histórica que vive Colombia señala que este planteamiento no desborda el espacio propio de la reflexión desde la psicología, sino que propone otros elementos a la representación del discurso social para favorecer las rupturas de lugares comunes y permitir la construcción de otras representaciones de lo real que puedan contribuir a modificar apreciaciones y comportamientos (Colmenares, 2002a, p. 17).

La definición que aporta María Eugenia Colmenares (2002b) sobre la resiliencia, tiene valor en la medida en que pone a dialogar el concepto con el compromiso ético y político de los sujetos que afrontan la adversidad en búsqueda de sentidos de vida y transformaciones históricas.

Llamamos resiliencia a la capacidad que muestran algunos seres humanos, en medio de las peores adversidades, de tomar el contrapié a partir de sí mismos y que les permite desarrollarse y construir sus sueños conservando la estructura humana en términos de valores éticos de supervivencia (Colmenares, 2002b, p. 22).

María Stella Rodríguez-Arenas (2004) hace un interesante trabajo al poner en diálogo la resiliencia con el desplazamiento forzado desde las vivencias de niños víctimas del conflicto armado. A partir de una alternativa pedagógica que tiene en la palabra su fuente 
de inspiración, permite recuperar al sujeto como actor de su proyecto de vida, emergiendo visiones prospectivas, ideales, sueños e ilusiones, que de alguna manera manifiestan cualidades resilientes, expresadas en la capacidad de estos niños, para convertir su experiencia de vida en narración, que les permita visualizar las experiencias difíciles como una parábola, con la posibilidad de crear un final feliz.

La resiliencia no resulta solo de lo individual o lo colectivo, sino que deriva del interjuego de ambos aspectos. El punto de encuentro de estas dos posiciones radica en que es una posibilidad del ser humano de resignificar la adversidad en términos positivos... pensamos que esta capacidad como muchas de las facultades humanas no es totalmente innata, ni totalmente adquirida más bien requiere de unas condiciones particulares que permitan que las personas puedan responder con esta fortaleza a las dificultades de la vida (Rodríguez-Arenas, 2004, p. 78).

Los trabajos de Aldo Melillo y Elbio Néstor Suárez-Ojeda (2002) y de estos con Daniel Rodríguez (2004) posicionaron una concepción latinoamericana de la resiliencia en situaciones de exclusión y de pobreza, que acentúa su dimensión comunitaria y la significación que para estas situaciones tienen los procesos resilientes. Tales perspectivas se distanciaban de la mirada clásica de los primeros estudios en resiliencia, cuyo interés se concentraba en los atributos individuales y en la relación de estos con los entornos sociales, y en reconocer el poder de la resiliencia para luchar contra las inequidades de la sociedad actual:

A partir de este enfoque, la resiliencia comunitaria desplaza la base epistemológica del concepto inicial, modificando no solo el objeto de estudio, sino también la postura del observador y los criterios de observación y validación del fenómeno... Cada desastre o catástrofe que una comunidad sufre representa un daño en términos de pérdidas de recursos y de vidas... Esa desgracia puede significar el desafío para movilizar las capacidades solidarias de la población y emprender procesos de renovación, que modernicen no solo la estructura física sino toda la trama social en esa comunidad (Suárez, 2002, pp. 70-71).

La perspectiva que aporta Elbio Néstor Suárez-Ojeda (2002) permite entonces pensar la posibilidad de una "teoría latinoamericana de la resiliencia, con enfoques más adecuados a esta realidad social" (Suárez, 2002, p. 67) que se sabe imbricada entre los problemas de una sociedad inequitativa, excluyente e injusta y los desafíos que estas realidades plantean a la misma resiliencia y su capacidad para potenciar respuestas que permitan fortalecer las dinámicas de agenciamiento y liberación de los sujetos y las comunidades y sus prácticas de resistencia y liberación frente a poderes que estructuralmente violentan la dignidad humana.

En su trabajo, después de reconocer el avance y posicionamiento de la resiliencia en América Latina, Elbio Néstor Suárez-Ojeda (2002) presenta algunos pilares de lo que él Ilama resiliencia comunitaria. Tales pilares son: "la autoestima colectiva, la identidad cultural, el humor social y la honestidad estatal" (Suárez-Ojeda, 2002, p. 72).

De la autoestima colectiva se destacan la actitud y el sentimiento de orgullo por el lugar en que se vive, el aprecio por la geografía, el clima y las tradiciones culturales. Se trata de un reconocimiento de lo que se es, de los orígenes y de las herencias recibidas de los antepasados. No es extraño que aquellas ciudades o localidades en las que se observa 
una elevada autoestima y orgullo de sus tradiciones tengan mayor capacidad de recuperación frente a la adversidad.

Por identidad cultural se reconoce esa persistencia del ser social en su unidad y "mismidad" por medio de cambios y circunstancias diversas. Tiene qué ver con la valoración de los rasgos culturales que nos develan sentido de pertenencia a un territorio y una cultura propia y singular. De allí que aquellas poblaciones que han hecho un baluarte del respeto y la exaltación de sus culturas tradicionales hayan mostrado una mayor capacidad para recomponerse y renacer luego de numerosas adversidades.

Otra variable importante en la resiliencia es el humor, que ha merecido un amplio estudio. El humor social como una capacidad propia de la idiosincrasia de personas y grupos de rasgos culturales particulares se potencia al encontrar la "comedia" en la propia tragedia, que se apoya en la flexibilidad y la relajación que puede brindar cierto sentido del humor, en medio de la complejidad de las situaciones, lo cual favorece la capacidad para hallar respuestas originales y soluciones innovadoras en medio de la crisis.

Un último componente es el designado como honestidad colectiva o estatal. Más allá de la transparencia en el manejo de la cosa pública, esto implica la existencia de una conciencia colectiva que desaprueba la deshonestidad de los funcionarios públicos y que valora un ejercicio decente de la gestión estatal, al reconocer su esfuerzo por respetar los derechos de los ciudadanos. Se trata de una intolerancia activa frente a la corrupción que se ha venido instalando en muchos niveles de la sociedad, tanto en la esfera pública como en la privada.

Otros asuntos como la capacidad de generar liderazgos auténticos y participativos, el ejercicio de una democracia efectiva en la toma de decisiones, en las que se reconozca la diversidad y no haya discriminación, fortalecen también el proceso de la resiliencia comunitaria. En contraste con estos pilares, desde la perspectiva de Suárez-Ojeda (2002), están los "antipilares".

Como antípodas de estos pilares, se han podido detectar condiciones o características que reducen la resiliencia comunitaria o, dicho de otra manera, inhiben la capacidad solidaria de reacción frente a la adversidad colectiva. Entre estos "antipilares" cabe mencionar: malinchismo, fatalismo, autoritarismo, corrupción (Suárez-Ojeda, 2002, p. 76).

Las comunidades combinan pilares y "antipilares" y de su reconocimiento se podrán elaborar predicciones, que ayuden a orientar y fortalecer la intervención social para poder dinamizar la resiliencia colectiva o comunitaria.
Este desplazamiento de las investigaciones centradas en el sujeto herido que afronta la adversidad y en las comunidades sometidas a tensiones y conflictos sociales complejos, se fue abriendo camino, no sin la preocupación que manifestaban algunos académicos por el mismo concepto de resiliencia, su significación y sus usos en algunos programas de atención e intervención a la infancia y adolescencia (Llobet \& Wegsman, 2004).

Valeria Llobet (2005) más adelante afirma que el mismo concepto puede ser mal interpretado y usado de manera tendenciosa, desde su asociación con intervenciones pobres para pobres, pasando por la promoción de un conformismo con connotaciones conservadoras. Y considera que hay una línea de problemas teóricos, políticos y éticos, cuyo debate según la autora no ha sido saldado entre los investigadores en resiliencia.

Es sencillo suponer que, o bien es posible predicar de alguien que "es" resiliente por sus peculiares características personales (inteligencia, astucia, etc.) lo que sería una aproximación psicologista y objetivizante, o que en realidad el ascenso social depende de las mismas capacidades por las cuales alguien puede lograr que la adversidad no se transforme en destino (Llobet, 2005, p. 13).

Frente a esta crítica que se deja planteada, la autora reconoce que la resiliencia puede tener una utilidad científica y que en medio de sus tensiones teóricas y de intervención, puede ser significativa para explicar problemas de vida, al favorecer la apertura hacia caminos creativos de reconstrucción personal y social.

La resiliencia, entonces, no es un rasgo de personalidad, sino que las personas son actores y fuentes de las actuaciones resilientes, y las familias, escuelas, comunidades, servicios sociales son el escenario de promoción de la resiliencia, y propician el despliegue, proveen de los factores protectores... redefinimos el concepto como procesos resilientes, entendiéndolos como la posibilidad de protección y autonomía frente a situaciones de adversidad apoyados en el proceso de subjetivación (Llobet, 2005, pp. 14-15).

Según Valeria Llobet (2005) es la interfase entre los niveles individuales, comunitarios y sus interacciones, lo que hace más interesante el concepto y lo que permite reconocer la potencia de la resiliencia, para abandonar un determinismo fatal, encontrando en ella una posibilidad para recuperar una visión de la micropolítica como espacio para la resistencia a la reproducción y las dinámicas del poder, que tiende a hegemonizar a los sujetos y las comunidades para que escriban una única historia, lo que les impide la creatividad para escribir su propio relato. La resiliencia está 
en esas márgenes y en las líneas de fuga que permiten a los sujetos y las comunidades ser actores de su propia construcción histórica.

Desde una mirada social, Joseba Azkarraga (2014) se refiere a la resiliencia en su aspecto local y comunitario, la pone en tensión frente a la crisis sistémica del planeta, y reconoce que es necesario hacerle preguntas a un mundo excesivamente consumidor que produce más residuos de los que puede absorber. Estamos — según Joseba Azkarraga (2014) — en una fase de translimitación, donde se han superado los límites impuestos por la naturaleza. Desde su perspectiva, la resiliencia se puede ubicar en la búsqueda de un cambio medioambiental global, como iniciativas regeneradoras de territorios más resilientes.

Por ello hablamos de la necesidad de construir resiliencia (familiar, organizativa, comunitaria, nacional), entendida como la capacidad de un sistema de absorber los choques o presiones externas y de reorganizarse, sin perder su estructura, función e identidad esencial (Azkarraga, 2014, pp. 92-93).

Esta manera de entender la resiliencia deja abierta la posibilidad de poner en diálogo las ciencias sociales con las ciencias naturales pues entiende que la crisis global es una crisis socioecológica, que está reclamando soluciones integradoras en cuanto a una auténtica transición hacia la sostenibilidad responsable del planeta.

El aporte latinoamericano propone un desplazamiento de la mirada más centrada en lo individual de los sujetos, hacia el ámbito de lo social, donde acontecen los procesos de interacción y de construcción del tejido comunitario, de participación democrática y acción política. La resiliencia comunitaria implica una conciencia de responsabilidad común frente a lo que afecta al sujeto como colectivo. La resiliencia comunitaria permite explorar las fronteras donde la resiliencia se encuentra con la política. Desde este horizonte comprensivo, se deben reconocer los esfuerzos de los investigadores por enfrentar diversas problemáticas sociales desde la mirada resiliente.

El trabajo de Patricia Granada-Echeverry y Sara Victoria Alvarado (2010) se centró precisamente en la relación entre resiliencia y política, y buscaba mostrar la contribución de la resiliencia en el fortalecimiento de la subjetivación política de niños y jóvenes que, en situación de adversidad, son capaces de agenciar experiencias vitales, desde la construcción de narrativas que les permiten actuar políticamente en sus vidas y sus entornos. Lo novedoso de este acercamiento es el de haber puesto en la mesa de la discusión la dimensión sociopolítica de la resiliencia, o la significación de la resiliencia en el horizonte de lo político que acontece en la vida de la gente.

Propusimos en este estudio la definición sociopolítica de la resiliencia como: la conservación, despliegue y desarrollo de las dimensiones humanas en contextos de adversidad, entre los que se destacan la dimensión crítica y la dimensión política que no se subordinan a la sobrevivencia, sino que, por el contrario, se transforman en discurso político, en posiciones de denuncia y de acción social transformadora ante el mundo. Esta definición sociopolítica de la resiliencia exigió redefinir el concepto de adversidad a partir de significados que incluyeran los significantes culturales, como las situaciones vividas cuya significación social estuviera ligada al sufrimiento humano como experiencia vital que hunde sus raíces en los mitos tanto de lo sacro como de lo profano que se reproducen en las acciones de protección y vulneración que tienen por sujeto/objeto a los niños y niñas más vulnerables (Granada-Echeverry \& Alvarado, 2010, p. 314). 
Lo novedoso de su acercamiento fue concebir los casos ejemplarizantes no como sujetos considerados "resilientes" desde la teoría clásica de la resiliencia, sino como ella lo afirma: "sujetos que están resiliando, es decir, "están-siendo" en la adversidad" (Granada-Echeverry \& Alvarado, 2010, p. 315). De esta forma, se buscó abrir la discusión al pensar la resiliencia no como una condición o una característica esperada de un sujeto frente a situaciones de adversidad, para entenderla mejor, sino como un proceso fenomenológico existencial mediante el cual el sujeto se constituye.

El término resiliencia emerge en esta investigación para dar cuenta, específicamente, del conjunto de estrategias vinculantes que llevan a cabo los niños, niñas y jóvenes en situación de calle para reparar el vínculo enfermo, enfrentar y resistir la adversidad y desarrollarse humanamente, logrando su inclusión progresiva a los bienes y servicios sociales, así como el ejercicio de su ciudadanía dentro de un proyecto de vida que incluye no solo su propia reivindicación sino la de otros en situaciones de vida semejantes a las suyas. Mediante el vínculo, el sujeto encuentra en el otro el reconocimiento y las oportunidades para lograr una parcela de dominio de sí mismo, de posesión de sí mismo, de placer de sí mismo, a partir del cual es posible la interacción con el otro, con el cuidado del otro y del mundo (GranadaEcheverry \& Alvarado, 2010, p. 322).

El trabajo de Granada y Alvarado (2010) se inscribe en un amplio espectro de ejercicios reflexivos, que tienen una marcada sensibilidad por comprender las transiciones que viven los sujetos y las comunidades al afrontar adversidades que surgen en contextos de violencia y exclusión. Estos trabajos expresan lo que se podría llamar la dimensión política de la resiliencia. Mencionemos algunos más significativos: Resiliencia y violencia política en niños (Silva, 1999a); Resiliencia y violencia política: la esperanza realista en el siglo XXI (Silva, 1999b); Factores psicosociales asociados con la resiliencia en niños colombianos víctimas de violencia intrafamiliar (Amar-Amar, Kotliarenco \& Abello-Llanos, 2003); Evaluación de factores de resiliencia en niños argentinos en condición de vulnerabilidad familiar (Morelato, 2014); Factores de resiliencia asociados al rendimiento académico en estudiantes de contextos de alta vulnerabilidad social (Villalta-Páucar, 2010); Factores que influyen en el pronóstico de recuperación de las familias en riesgo psicosocial: el papel de la resiliencia del menor (RodrigoLópez, Camacho-Rosales, Máiquez-Chávez, Byrne \& Benito-Cruz, 2009); Estudio de factores resilientes en familiares de personas con discapacidad (Ponce-Espino \& Torrecillas-Martín, 2014); Madres y padres en contextos de riesgo psicosocial (Martín, Cabrera, León \& Rodrigo-López, 2013); Construcción de escala de factores personales de resiliencia en mujeres víctimas de desplazamiento forzado (Amar-Amar, Utria-Utria, Abello-Llanos, Martínez-González \& Crespo-Romero, 2014); Resiliencia cultural comunitaria como quehacer político femenino de las mujeres williche del Chaurakawin, Región de los Lagos, Chile (Duquesnoy, 2014); Un modelo teórico de la resiliencia familiar en contextos de desplazamiento forzado (Domínguez de la Ossa, 2014); Transversalidad y resiliencia en los relatos de desplazados del Oriente de la Zona Metropolitana de la Ciudad de México (Domínguez-Echeverría \& Espinosa-García, 2017).

Los ejercicios reflexivos tomados como ejemplo muestran la pertinencia de la resiliencia para la promoción de una salud integral y la recuperación del tejido humano y social; abren la pregunta por las posibilidades de una existencia digna del sujeto en contextos de marginalidad, violencias y poderes de dominación. Es necesario que la resiliencia se plantee 
su responsabilidad ética y política, que implica ir más allá del afrontamiento o la resistencia frente a la adversidad, hacia un compromiso con las transformaciones históricas necesarias para una auténtica humanización de la sociedad. Esta convicción deja instalada la pregunta por la dimensión política de la resiliencia y su significación en el ámbito de la acción política.

\section{Conclusiones}

Las diversas definiciones sobre resiliencia muestran la proliferación de acercamientos a la categoría, como su complejidad conceptual al entenderla como capacidad, proceso, rasgo, adaptación positiva, habilidad, potencialidad, paradigma, cualidad y estrategia de afrontamiento de la adversidad. Llama la atención la primacía de la comprensión psicológica y el acentuado carácter de la respuesta más individual de los sujetos frente a la adversidad. Este enfoque, si bien reconoce los procesos de interacción y los vínculos de apoyo, concentra la eficacia del proceso resiliente, en las posibilidades del sujeto y su adaptabilidad en medio de situaciones de adversidad.

La perspectiva de la resiliencia comunitaria permite reconocer el valor de las redes humanas, la cultura y las comunidades locales, en el fortalecimiento y el florecimiento de una vida digna. En su puesta en escena, no solo favorece la mitigación del impacto de las condiciones sociales y ambientales adversas, sino que abre la posibilidad para el posicionamiento del concepto de resiliencia política, al ser puente entre las acciones de los sujetos por el reconocimiento y las acciones colectivas que jalonan prácticas humanas de agenciamiento y liberación.

Se hace necesario pasar del sujeto individual al sujeto histórico, un sujeto social, cultural y político, que permita plantearse la pregunta por la relación entre resiliencia y política. Un estudio de la resiliencia, desde la dimensión más comunitaria y social, ayudaría a deslocalizar al sujeto individual, para poder entender la resiliencia como un proceso humano histórico que acontece en sujetos plurales, con autonomía, conciencia histórica, capacidad ética y estética para imaginar, desde otros y con otros, un mundo donde sea posible reinventarnos para la vida.

\section{Sobre los autores}

Luis Fernando Granados-Ospina es filósofo de la Pontificia Universidad Javeriana. Licenciado en teología, Pontificia Universidad Javeriana. Licenciado en pedagogía, Pontificia Universidad de Comillas (España). Magíster en educación, Pontificia Universidad Javeriana. Candidato a doctor en ciencias sociales, niñez y juventud del Centro de Estudios Avanzados en Niñez y Juventud (alianza CINDE-Universidad de Manizales).
Sara Victoria Alvarado-Salgado es psicóloga, Pontificia Universidad Javeriana. Magíster en ciencias del comportamiento y doctora en educación, Nova University-CINDE. Posdoctora en Ciencias Sociales, Niñez y Juventud, Universidad Católica de São Paulo, Universidad de Manizales, Centro de Estudios Avanzados en Niñez y Juventud, Consejo Latinoamericano de Ciencias Sociales, CINDE-CLACSO. Directora del Centro de Estudios Avanzados en Niñez y Juventud, Universidad de Manizales-CINDE y directora del doctorado en ciencias sociales, niñez y juventud. Coordinadora Red Iberoamericana de Posgrados en Infancia y Juventud, RedINJU. Integrante del comité académico del programa posdoctoral de investigación en ciencias sociales, niñez y juventud, y coordinadora académica de la Escuela Internacional de Posgrados en Infancia y Juventud. Gerente del programa de investigación cofinanciado por Colciencias: Sentidos y prácticas políticas de niños y jóvenes en torno a la democracia, la reconciliación y la paz. Actualmente, investigadora de las investigaciones Procesos de construcción social de la niñez en contextos de conflicto armado: la paz, la reconciliación y la democracia desde la perspectiva de narrativas generativas de niños y niñas y Narrativas colectivas de paz y conflicto armado desde las voces de los niños y niñas de la primera infancia, familias y agentes relacionales en el marco del posconflicto/ posacuerdo, del grupo de investigación Perspectivas políticas, éticas y morales de la niñez y la juventud (categoría A en Colciencias) adscrito al Centro de Estudios Avanzados en Niñez y Juventud, CINDE-Universidad de Manizales. Fundadora del programa Niños, Niñas y Jóvenes Constructores de la Paz, creado en 1998.

Jaime Carmona-Parra es posdoctorado, Universidad de Manizales, CINDE, Universidad Católica de São Paulo, CLACSO. Doctor en psicología social, Universidad Complutense de Madrid. Magíster en ciencias sociales, Universidad de Antioquia. Psicólogo, Universidad Autónoma Monterrey de Costa Rica. Profesor del doctorado en ciencias sociales, niñez y juventud, Universidad de Manizales, Centro de Estudios Avanzados en Niñez y Juventud, CINDE. Director de la Escuela de Psicología, Universidad de Manizales.

\section{Referencias}

Álvarez, Liliana H. \& Hurley, Dermot J. (2010). Desde la zona de riesgo a la zona de resiliencia. Revista Científica de UCES, Universidad de Ciencias Empresariales y Sociales, 14 (2), 13-36. Disponible en: http://dspace.uces.edu.ar:8180/xmlui/bits tream/handle/123456789/890/Desde_la_zona_ de_riesgo_Alvarez.pdf? sequence $=1$

Amar-Amar, José Juan; Kotliarenco, María Angélica \& Abello-Llanos, Raimundo (2003). Factores psicosociales asociados con la resiliencia en niños colombianos víctimas de violencia intrafamiliar. Investigación \& Desarrollo. Universidad del Norte, 11 (1), 162-197. Disponible en: http://rcientificas. uninorte.edu.co/index.php/investigacion/article/ view/1134/710 
Amar-Amar, José Juan; Utria-Utria, Leider Miguel; Abello-Llanos, Raimundo; Martínez-González, Marina Begoña \& Crespo-Romero, Fernando Alexis (2014). Construcción de la Escala de Factores Personales de Resiliencia (FPR-1) en mujeres víctimas del desplazamiento forzado en Colombia. Universitas Psychologica, 13 (3), 853-864. doi:10.11144/Javeriana. UPSY13-3.cefp. Disponible en: http://revistas.javeriana.edu.co/index. php/revPsycho/article/viewFile/4672/8730

Azkarraga, Joseba (2014). Resiliencia local y comunitaria frente a la crisis sistémica. En José María Madariaga (coord.). Nuevas miradas sobre la resiliencia: ampliando ámbitos y prácticas, 85-110. Barcelona: Gedisa.

Becoña, Elisardo (2006). Resiliencia: definición, características y utilidad del concepto. Revista de Psicopatología y Psicología Clínica, 11 (3), 125-146. Asociación Española de Psicología Clínica y Psicopatología, AEPCP. Disponible en: http://aepcp.net/arc/01.2006(3).Becona.pdf

Bowlby, John (1995). Vínculos afectivos: formación, desarrollo y pérdida. Madrid: Morata.

Campo-Vásquez, Rafael; Granados-Ospina, S.J., Luis Fernando; MuñozOrtega, Liliana; Rodríguez-Arenas, María Stella \& Trujillo-García, Sergio (2012). Caracterización del avance teórico, investigativo y/o de intervención en resiliencia desde el ámbito de las universidades en Colombia. Universitas Psychologica, 11 (2), 545-557. Disponible en: http://www. javeriana.edu.co/universitaspsychologica/articulo.php?art=854

Cicchetti, Dante (2003). Foreword. En Suniya S. Luthar (ed.). Resilience and Vulnerability: Adaptation in the Context of Childhood Adversities, xixxxvii. Cambridge, Reino Unido: Cambridge University Press.

Colmenares, María Eugenia (2002a). Introducción. En Esther Sánchez-Botero \& Ana Claudia Delgado (dirs). La resiliencia. Responsabilidad del sujeto y esperanza social, 15-18. Cali. Casa Editorial Rafue.

Colmenares, María Eugenia (2002b). Resiliencia: sus valores psicológicos y socioculturales. En Esther Sánchez-Botero \& Ana Claudia Delgado (dirs). La resiliencia. Responsabilidad del sujeto y esperanza social, 21-46. Cali. Casa Editorial Rafue.

Cyrulnik, Boris (2001). La maravilla del dolor: el sentido de la resiliencia. Barcelona: Granica.

Cyrulnik, Boris (2005a). Bajo el signo del vínculo: una historia natural de apego. Barcelona: Gedisa.

Cyrulnik, Boris (2005b). El amor que nos cura. Madrid: Gedisa.

Cyrulnik, Boris (2014). La resiliencia en el siglo XXI, entrevista a Boris Cyrulnik. En José María Madariaga (coord.). Nuevas miradas sobre la resiliencia: ampliando ámbitos y prácticas, 31-51. Barcelona: Gedisa.

Cyrulnik, Boris; Manciaux, Michel; Sánchez-Botero, Esther; Colmenares, María Eugenia; Olaya, María Margarita \& Balegno, Lorenzo (2002). La resiliencia. Desvictimizar la víctima. Cali: Casa Editorial Rafue.

Cyrulnik, Boris; Manciaux, Michel; Vanistendael, Stefan \& Lecomte, Jacques (2003). La resiliencia: estado de la cuestión. En Michel Manciaux (comp.). La resiliencia: resistir y rehacerse, 17-28. Barcelona: Gedisa.

Cyrulnik, Boris; Tomkiewicz, Stanislaw; Guénard, Tim; Vanistendael, Stefan; Manciaux, Michel \& Balegno, Lorenzo (2004). El realismo de la esperanza. Testimonios de experiencias profesionales en torno a la resiliencia. Barcelona: Gedisa.

Delage, Michel (2010). La resiliencia familiar. Barcelona: Gedisa.

Domínguez de la Ossa, Elsy Mercedes (2014). Un modelo teórico de la resiliencia familiar en contextos de desplazamiento forzado. (Tesis doctoral. Centro de Estudios Avanzados en Niñez y Juventud, CINDE, alianza de la Universidad de Manizales y el CINDE, Manizales). Disponible en: 
http://biblioteca.clacso.edu.ar/Colombia/alian za-cinde-umz/20140910011354/Elsymercedes dominguez.pdf

Domínguez-Echeverría, María Patricia \& EspinosaGarcía, Santiago (2017). Transversalidad y resiliencia en los relatos de desplazados del Oriente de la Zona Metropolitana de la Ciudad de México. Saskab. Revista de Discusiones Filosóficas Desde Acá, Cuaderno 10, 1-17. Disponible en: http://www. ideaz-institute.com/sp/CUADERNO10/C101.pdf

Duquesnoy, Michel (2014). Resiliencia cultural comunitaria como quehacer político femenino de las mujeres williche del Chaurakawin (Región de los Lagos, Chile). Cuicuilco, Revista de la Escuela Nacional de Antropología e Historia, México, 59, 65-91. Disponible en: http://www.redalyc.org/ pdf/351/35131858004.pdf

Forés, Anna \& Grané, Jordi (2008). La resiliencia. Crecer desde la adversidad. Madrid: Narcea. Barcelona: Plataforma Editorial.

Forés, Anna \& Grané, Jordi (2012). La resiliencia en entornos socioeducativos. Madrid: Narcea.

García-Vesga, María Cristina \& Domínguez de la Ossa, Elsy Mercedes (2013). Desarrollo teórico de la resiliencia y sus aplicaciones en situaciones de adversidad: una revisión analítica. Revista Latinoamericana de Ciencias Sociales, Niñez y Juventud, 11 (1), 63-77. Disponible en: http:// biblioteca.clacso.edu.ar/Colombia/alianza-cin de-umz/20130717011954/RevistaLatinoamerica naVol.11N.1.pdf

Garmezy, Norman (1991). Resilience in Children's Adaptation to Negative Life Events and Stressed Environments. Pediatric Annals, 20 (9), 459-466.

Gómez, Esteban \& Kotliarenco, María Angélica (2010). Resiliencia familiar: un enfoque de investigación e intervención con familias multiproblemáticas. Revista de Psicología, 19 (2), 103-132 (agosto-diciembre, 2010). Santiago de Chile. Universidad de Chile. Disponible en: http://repo sitorio.uchile.cl/bitstream/handle/2250/129448/ Resiliencia-familia-un-enfoque-de-investiga cion-e-intervencion-con-familias-multiproble maticas. pdf? sequence $=1$ \&isAllowed $=y$

Granada-Echeverry, Patricia \& Alvarado, Sara Victoria (2010). Resiliencia y sentido político en niños y niñas en situación de calle. Revista Latinoamérica de Ciencias Sociales, Niñez y Juventud, 8 (1), 311-327. Disponible en: http://biblio teca.clacso.edu.ar/Colombia/alianza-cindeumz/20130712043955/ArtPatriciaGranadaEche verry.pdf

Henderson-Grotberg, Edith (2006). La resiliencia en el mundo de hoy: cómo superar las adversidades. Barcelona: Gedisa.
Henderson, Nan \& Milstein, Mike M. (2003). Resiliencia en la escuela. Barcelona: Paidós.

Infante-Espínola, Francisca (2002). La resiliencia como proceso: una revisión de la literatura reciente. En Aldo Melillo \& Elbio Néstor Suárez-Ojeda (comps.). Resiliencia: descubriendo las propias fortalezas, 31-53. Buenos Aires: Paidós.

Kalawski, Juan Pablo \& Haz, Ana María (2003). Y... ¿Dónde está la resiliencia? Una reflexión conceptual. Interamerican Journal of Psychology, 37 (2), 365-372. Disponible en: http://www.redalyc. org/articulo.oa?id=28437213

Kotliarenco, María Angélica (2014). CEANIM: 34 años de historia de resiliencia comunitaria. En José María Madariaga (coord.). Nuevas miradas sobre la resiliencia: ampliando ámbitos y prácticas, 153-171. Barcelona: Gedisa.

Kotliarenco, María Angélica; Cáceres, Irma \& FonteciIla, Marcelo (1997). Estado del arte en resiliencia. Washington: Organización Panamericana de la Salud. Disponible en: http://www1.paho.org/hq/ dmdocuments/2009/Resil6x9.pdf

Kotliarenco, María Angélica; Mardones, Francisco; Melillo, Aldo \& Suárez-Ojeda, Elbio Néstor (2000). Actualizaciones en resiliencia. Buenos Aires: Fundación Bernard van Leer, Colección Salud Comunitaria, Serie Resiliencia, Ediciones de la Universidad Nacional de Lanús, UNLa.

Llobet, Valeria (2005). La promoción de la resiliencia con niños y adolescentes: entre la vulnerabilidad y la exclusión. Herramientas para la transformación. Buenos Aires: Novedades Educativas, Noveduc.

Llobet, Valeria \& Wegsman, Susana (2004). El enfoque de resiliencia en los proyectos sociales: perspectivas y desafíos. Revista de Psicología de la Universidad de Chile, XIII (1), 143-152. Disponible en: https://www.aacademica.org/valeria.Ilo bet/63.pdf

Madariaga, José María (coord.) (2014). Nuevas miradas sobre la resiliencia: ampliando ámbitos y prácticas. Barcelona: Gedisa.

Madariaga, José María; Palma-García, María de las Olas; Surjo, Pilar; Villalba, Cristina \& Arribillaga, Ana (2014). La construcción social de la resiliencia. En José María Madariaga. Nuevas miradas sobre la resiliencia: ampliando ámbitos y prácticas, 11-30. Barcelona: Gedisa.

Manciaux, Michel (comp.) (2003). La resiliencia: resistir y rehacerse. Barcelona: Gedisa.

Martín, Juan Carlos; Cabrera, Eduardo, León, Jaime \& Rodrigo-López, María José (2013). La escala de competencia y resiliencia parental para madres y padres en contextos de riesgo psicosocial. Anales de Psicología, Universidad de Murcia, España, 
29 (3), 886-896. Disponible en: http://www.re dalyc.org/pdf/167/16728244028.pdf

Masten, Ann S. (1994). Resilience in Individual Development: Successful Adaptation despite Risk and Adversity. En Margaret C. Wang \& Edmund W. Gordon (eds.). Educational Resilience in InnerCity America: Challenges and Prospects, 3-25. Hillsdale, New Jersey: Lawrence Erlbaum.

Melillo, Aldo; Estamatti, Mirta \& Cuestas, Alicia (2000). Algunos fundamentos psicológicos del concepto de resiliencia. En María Angélica Kotliarenco, Francisco Mardones, Aldo Melillo \& Elbio Néstor Suárez-Ojeda. Actualizaciones en resiliencia, 83102. Buenos Aires: Fundación Bernard van Leer, Colección Salud Comunitaria, Serie Resiliencia, Ediciones de la Universidad Nacional de Lanús, UNLa.

Melillo, Aldo \& Suárez-Ojeda, Elbio Néstor (comps.) (2002). Resiliencia: descubriendo las propias fortalezas. Buenos Aires: Paidós.

Melillo, Aldo; Suárez-Ojeda, Elbio Néstor \& Rodríguez, Daniel (comps.) (2004). Resiliencia y subjetividad: los ciclos de la vida. Buenos Aires: Paidós.

Morelato, Gabriela Susana (2014). Evaluación de factores de resiliencia en niños argentinos en condiciones de vulnerabilidad familiar. Universitas Psychologica, 13 (4), 15-30. Disponible en: http:// revistas.javeriana.edu.co/index.php/revPsycho/ article/view/5295/9857

Ponce-Espino, Jordi Eusebio \& Torrecillas-Martín, Ana María (2014). Estudio de factores resilientes en familiares de personas con discapacidad. Revista INFAD de Psicología, International Journal of Developmental and Educational Psychology, 6 (1), 431-442. Disponible en: http://www.infad. eu/RevistaINFAD/OJS/index.php/IJODAEP/article/ view/763/699

Pourtois, Jean Pierre (2014). Los recursos de la resiliencia. En José María Madariaga (coord.). Nuevas miradas sobre la resiliencia: ampliando ámbitos y prácticas, 69-83. Barcelona: Gedisa.

Puig, Gema \& Rubio, José Luis (2011). Manual de resiliencia aplicada. Barcelona: Gedisa.

Puig, Gema \& Rubio, José Luis (2015). Tutores de resiliencia: dame un punto de apoyo y moveré MI mundo. Barcelona: Gedisa.

Real Academia Española, RAE (2014). Diccionario de la Lengua Española. Vigesimotercera Edición: Madrid. Espasa.

Rodrigo-López, María José; Camacho-Rosales, Juan; Máiquez-Chávez, María Luisa; Byrne, Sonia \& Benito-Cruz, José Manuel (2009). Factores que influyen en el pronóstico de recuperación de las familias en riesgo psicosocial: el papel de la resiliencia del menor. Psicothema, Universidad de la Laguna, 21 (1), 90-96. Disponible en: http:// www.psicothema.es/pdf/3600.pdf

Rodríguez-Arenas, María Stella (2004). Resiliencia: otra manera de ver la adversidad. Alternativa pedagógica para la atención de niños y niñas en situación de desplazamiento. Bogotá: Colección Fe y Universidad, Pontificia Universidad Javeriana.

Rutter, Michael (1993). Resilience: Some Conceptual Considerations. Journal of Adolescent Health, 14 (8), 626-631. Disponible en: http://www.jahonli ne.org/article/1054-139X(93)90196-V/pdf

Rutter, Michael (2012). Resilience as a Dynamic Concept. Development and Psychopathology, 24 (2), 335-344.

Silva, Giselle (1999a). Resiliencia y violencia política: la esperanza realista en el siglo XXI. En María Rosa Fort-Brescia \& Moisés Lemlij (eds.). El umbral del milenio, 71-94. Lima: Comisión de Promoción del Perú, Seminario Interdisciplinario de Estudios Andinos, SIDEA.

Silva, Giselle (1999b). Resiliencia y violencia política en niños. Buenos Aires: Universidad Nacional de Lanús, UNLa. Fundación Bernard van Leer, Colección Salud Comunitaria, Serie Resiliencia.

Suárez-Ojeda, Elbio Néstor (2002). Una concepción latinoamericana: la resiliencia comunitaria. En Aldo Melillo \& Elbio Néstor Suárez-Ojeda (comps.). Resiliencia descubriendo las propias fortalezas, 6782. Buenos Aires: Paidós.

Vanistendael, Stefan (2014). Resiliencia: el reto del cambio de mirada. En José María Madariaga (coord.). Nuevas miradas sobre la resiliencia: ampliando ámbitos y prácticas. Barcelona: Gedisa.

Vanistendael, Stefan \& Lecomte, Jacques (2002). La felicidad es posible: despertar en niños maltratados la confianza en sí mismos. Construir la resiliencia. Barcelona: Gedisa.

Villalta-Páucar, Marco Antonio (2010). Factores de resiliencia asociados al rendimiento académico en estudiantes de contextos de alta vulnerabilidad social. Revista de Pedagogía, Caracas, Escuela de Educación, Universidad Central de Venezuela, 31 (88), 159-188. Disponible en: http://www.re dalyc.org/pdf/659/65916617007.pdf

Walsh, Froma (2004). Resiliencia familiar: estrategias para su fortalecimiento. Buenos Aires: Amorrortu.

Werner, Emmy E. \& Smith, Ruth S. (1982). Vulnerable but Invincible: A Study of Resilient Children. New York: McGraw-Hill.

Werner, Emmy E. \& Smith, Ruth S. (1992). Overcoming the Odds: High Risk Children from Birth to Adulthood. Ithaca, New York: McGraw-Hill.

Werner, Emmy E. \& Smith, Ruth S. (2001). Journeys from Childhood to Midlife. Risk, Resilience, and Recovery. Ithaca, New York: Cornell University Press. 